\title{
Polymer and Carbon Spheres with an Embedded Shell of Plasmonic Gold Nanoparticles
}

Mostofa K. Khan and Mark J. MacLachlan*

Department of Chemistry, University of British Columbia, 2036 Main Mall, Vancouver, BC V6T 1Z1 CANADA. mmaclach@chem.ubc.ca

Supporting Information 


\section{Experimental}

Materials. 3-Aminophenol (98\%, Aldrich), formaldehyde (37\%, Fisher Scientific), hydrogen tetrachloroaurate(III) hydrate (99.9\% trace metal basis, Aldrich), ammonium hydroxide (28\%, Fisher Scientific), and 4-amino-3-hydrazino-5-mercapto-1,2,4-triazole (also known as Purpald $₫$, $\geq 99 \%$, Aldrich) were purchased from respective commercial sources and used as received without purification.

Characterization Techniques. Scanning electron microscopy (SEM) images with backscattered electron (BSE) detector were collected using a Hitachi S4700 electron microscope on samples sputter-coated with carbon prior to imaging. All other SEM images were captured with secondary electron (SE) detector using the same microscope on samples sputter-coated with $\mathrm{Pt} / \mathrm{Pd}(80 / 20)$ alloy. All samples for SEM imaging were prepared by drop casting the aqueous dispersions on aluminum stubs and drying at ambient conditions prior to sputter-coating. Samples for transmission electron microscopy (TEM) were prepared by drop casting the ethanolic dispersions on carbon-coated 400 mesh size TEM grids and drying at ambient conditions. TEM images of most of the samples were recorded with a Hitachi H7600 microscope operating at $80-100 \mathrm{kV}$. TEM images for tomography were recorded with a FEI Tecnai G20 200kV LaB6 microscope with FEI Eagle 4k Camera. Data were acquired using FEI's automated tomography software with a tilt series of -60 to +60 degrees. The individual tilt series images were aligned with a filtered cross-correlation algorithm in FEI's Inspect 3D reconstruction software. Raw data was then reconstructed using FEI Inspect 3D software and the Serial Iterative Reconstruction Technique (SIRT). 3D modeling was done with Amira modeling software. Density profile measurements were carried out using Volocity (PerkinElmer) 3D image analysis software. Raman spectra were recorded using a Raman microspectrometer that fiber-couples an IPS $300 \mathrm{~mW} 785 \mathrm{~nm}$ diode laser to an Olympus BX-51 microscope. This system focuses backscattered light, collected by a $5 x$ single-lens objective onto a six-around-one fiber bundle. The bundle exit, arranged as a vertical fiber stack, forms a $100 \mu \mathrm{m}$ slit at the entrance of an Acton $300 \mathrm{~mm}$ monochromator equipped with a 600 groove $\mathrm{mm}^{-1}$ grating, and coupled to a Princeton instruments PIXIS back-illuminated CCD detector. A computer reads the CCD and bins the pixels in each column, $t$, from 1 to 1400 to form a spectrum of scattered-light intensity versus Raman shifted wavelength, spanning a vibrational frequency interval from 300 to 1800 $\mathrm{cm}^{-1}$. Infrared spectra were recorded in attenuated total reflection (ATR) mode on a PerkinElmer Frontier FTIR spectrophotometer equipped with Universal ATR sampling Accessory. Elemental analysis $(\mathrm{CNH})$ were obtained at the UBC Microanalytical Facility. UV-visible-near infrared spectra of the aqueous dispersion of the samples were obtained on a Cary $5000 \mathrm{UV}$-Vis/NIR spectrophotometer. Powder X-ray diffraction patterns were recorded on a Bruker D8 Advance diffractometer using $\mathrm{Cu}-\mathrm{K} \alpha$ radiation and a $\mathrm{NaI}$ scintillation detector. Differential scanning calorimetry analysis was performed on a PerkinElmer Diamond DSC using $12 \mathrm{mg}$ of sample at a scanning rate of $10{ }^{\circ} \mathrm{C} / \mathrm{min}$ under nitrogen flow. The calorimeter was calibrated for temperature and energy with indium $\left(T_{\mathrm{m}}=156.60{ }^{\circ} \mathrm{C}, \Delta H_{\mathrm{f}}=28.45 \mathrm{~J} / \mathrm{g}\right)$ and zinc $\left(T_{\mathrm{m}}=419.47{ }^{\circ} \mathrm{C}, \Delta H_{\mathrm{f}}=\right.$ $108.37 \mathrm{~J} / \mathrm{g}$ ) reference materials. Thermogravimetric analysis was performed on a PerkinElmer STA 6000 simultaneous thermal analyzer at a scan rate of $10^{\circ} \mathrm{C} / \mathrm{min}$ under nitrogen. Nitrogen adsorption data were collected at $77 \mathrm{~K}$ using a Micromeritics ASAP 2020 analyzer. Samples were degassed under vacuum for $12 \mathrm{~h}$ prior to analysis. 


\section{Synthesis}

\section{Preparation of 3-aminophenol-formaldehyde resin nanospheres (RNS)}

In a typical procedure, $0.1820 \mathrm{~g}(1.67 \mathrm{mmol})$ of 3 -aminophenol was dissolved in a water $(12$ $\mathrm{mL})$ /ethanol $(4.8 \mathrm{~mL})$ binary solvent mixture. To this mixture, $110 \mu \mathrm{L}$ of aqueous ammonia solution $\left(\mathrm{NH}_{4} \mathrm{OH}, 28 \%\right)$ was added and the mixture was magnetically stirred at $30{ }^{\circ} \mathrm{C}$ for $10 \mathrm{~min}$. Formaldehyde solution $(125 \mu \mathrm{L}, 37 \mathrm{wt} \%)$ was then added and the reaction mixture was stirred at $30{ }^{\circ} \mathrm{C}$. The resulting reaction mixture started becoming a white cloudy suspension within a minute and was stirred for an additional $4 \mathrm{~h}$ to give monodisperse resin spheres (RNS1). Other samples (RNS2-RNS4) with smaller size were synthesized by varying the concentration of the resin precursors in the reaction mixtures (see Table S1 for details). Synthesized resin spheres were purified with distilled water by repeated centrifugation at a speed of $4500 \mathrm{rpm}$ for $40 \mathrm{~min}$ and sonication for $10 \mathrm{~min}$. These repeated sonication and centrifugation cycles were performed at least 9 times to confirm removal of unreacted reagents.

Table S1. Synthesis conditions for RNS1 - RNS2.

\begin{tabular}{|l|c|c|c|c|c|c|}
\hline Sample & $\begin{array}{c}\mathbf{2 8 \%} \mathbf{~ N H}_{\mathbf{3}(\mathbf{a q})} \\
(\mathbf{m L})\end{array}$ & $\begin{array}{c}\mathbf{H}_{\mathbf{2}} \mathbf{O} \\
(\mathbf{m L})\end{array}$ & $\begin{array}{c}\text { Ethanol } \\
(\mathbf{m L})\end{array}$ & $\begin{array}{c}\text { 3-aminophenol } \\
\mathbf{( g )}\end{array}$ & $\begin{array}{c}\text { 37\% Formaldehyde } \\
(\mathbf{m L})\end{array}$ & $\begin{array}{c}\text { Sphere size } \\
(\mathbf{n m})\end{array}$ \\
\hline RNS1 & 0.110 & 12.0 & 4.8 & 0.182 & 0.25 & $541 \pm 29$ \\
\hline RNS2 & 0.275 & 30.0 & 12.0 & 0.182 & 0.25 & $307 \pm 13$ \\
\hline RNS3 & 0.550 & 60.0 & 24.0 & 0.182 & 0.25 & $210 \pm 10$ \\
\hline RNS4 & 1.100 & 120.0 & 48.0 & 0.182 & 0.25 & $118 \pm 3$ \\
\hline
\end{tabular}

Preparation of the composite nanospheres comprising 3-aminophenol-formaldehyde and gold nanoparticles (AuNPs@RNS)

In a typical procedure, $5 \mathrm{mg}$ of dry resin spheres (RNS1-RNS4) was dispersed in $10 \mathrm{~mL}$ of 1.0 $\mathrm{mM} \mathrm{HAuCl}_{4}$ (aq) solution by simple magnetic stirring at room temperature. After $2 \mathrm{~h}$, the resulting composite materials were purified by washing with distilled water with alternating centrifugation and sonication cycles at least for 3 times. Various other samples as described in the main text were prepared following the same procedure and by changing only the concentration of the $\mathrm{HAuCl}_{4}$ (aq) solution or reaction time, unless otherwise mentioned.

Preparation of the carbon nanospheres (CNS1) and composite gold nanoparticles@carbon nanospheres (AuNPs@CNS1)

Carbon nanospheres (CNS1) and composite gold nanoparticles@carbon nanospheres (AuNPs@CNS1) were obtained from RNS1 and AuNPs@RNS1 samples, respectively, by pyrolyzing under $\mathrm{N}_{2}$ atmosphere from room temperature to $100^{\circ} \mathrm{C}$ at a heating rate of $2{ }^{\circ} \mathrm{C} / \mathrm{min}$, staying at $100{ }^{\circ} \mathrm{C}$ for $2 \mathrm{~h}$, then heating to $600{ }^{\circ} \mathrm{C}$ at $2{ }^{\circ} \mathrm{C} / \mathrm{min}$. Samples were kept at $600{ }^{\circ} \mathrm{C}$ for 6 $\mathrm{h}$ and cooled down to room temperature under continuous $\mathrm{N}_{2}$ flow, resulting in the corresponding materials. 


\section{Additional Discussion about the Preparation of the Gold-containing Nanospheres.}

To use the resin spheres both as a reductant and host for gold nanoparticles, as-synthesized RNS1 sample without any purification was mixed with $\mathrm{HAuCl}_{4}$ in an aqueous dispersion with magnetic stirring at room temperature. Fig. S4 shows the TEM images of the resulting materials. From Fig. S4a, it is clearly seen that AuNPs indeed formed inside the resin nanospheres. However, a significant number of AuNPs are adsorbed to the outer surface. Moreover, a TEM image of the supernatant solution obtained from these preparations (Fig. S4b) shows polydisperse AuNPs, indicating ex-situ reduction of gold precursor by unreacted reagents, leading to the formation of AuNPs that remained in the solvent and are not attached to the resin spheres. Prevention of this ex-situ reduction of gold precursor was necessary to obtain clean materials. Hence, in all of our subsequent preparations, as-synthesized resin spheres were thoroughly washed with distilled water until the washings were proved to be free of unreacted reagents, confirmed by the Purpald test for formaldehyde (Fig. S5), ${ }^{\mathrm{S} 1, \mathrm{~S} 2}$ before adding the gold precursor.

We attempted to synthesize composite nanomaterials comprising of colloidal resin spheres and gold nanoparticles (AuNPs) by adding $\mathrm{HAuCl}_{4}(\mathrm{aq})$ to the reaction mixture along with resin precursors for simultaneous formation of gold nanoparticle cores and resin shells. A TEM image (Fig. S6) of the resulting nanospheres obtained after centrifugation shows few clusters of nanoparticles on the surface without any AuNPs inside the resin spheres. This implies that instead of core-shell composite particles, resin nanospheres and gold nanoparticles formed independently. Most of the AuNPs remained dispersed in the solvent and are lost during centrifugation. Only a few AuNPs remained adsorbed onto the surface. This process led to significantly larger resin spheres (average size is $\sim 840 \mathrm{~nm}$ ) compared to similar preparations without gold precursor. 


\section{Supporting Figures}
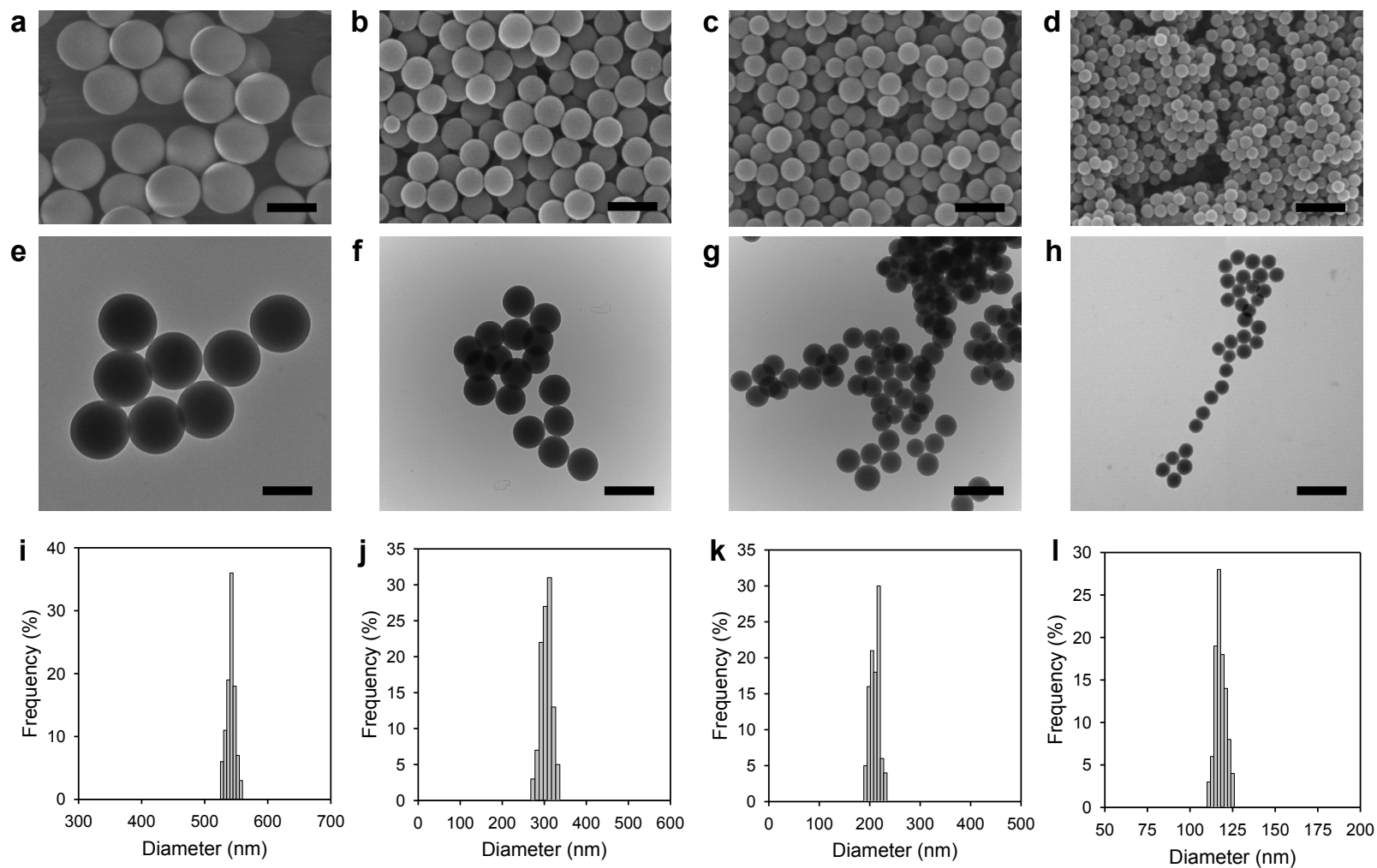

Figure S1. SEM images (first row), TEM images (second row) and size distribution histogram (third row) of RNS1 (a, e, i), RNS2 (b, f, j), RNS3 (c, g, k) and RNS4 (d, h, l) samples. Scale bars, $500 \mathrm{~nm}$. Approximately 100 independent particles were measured for each analysis. 


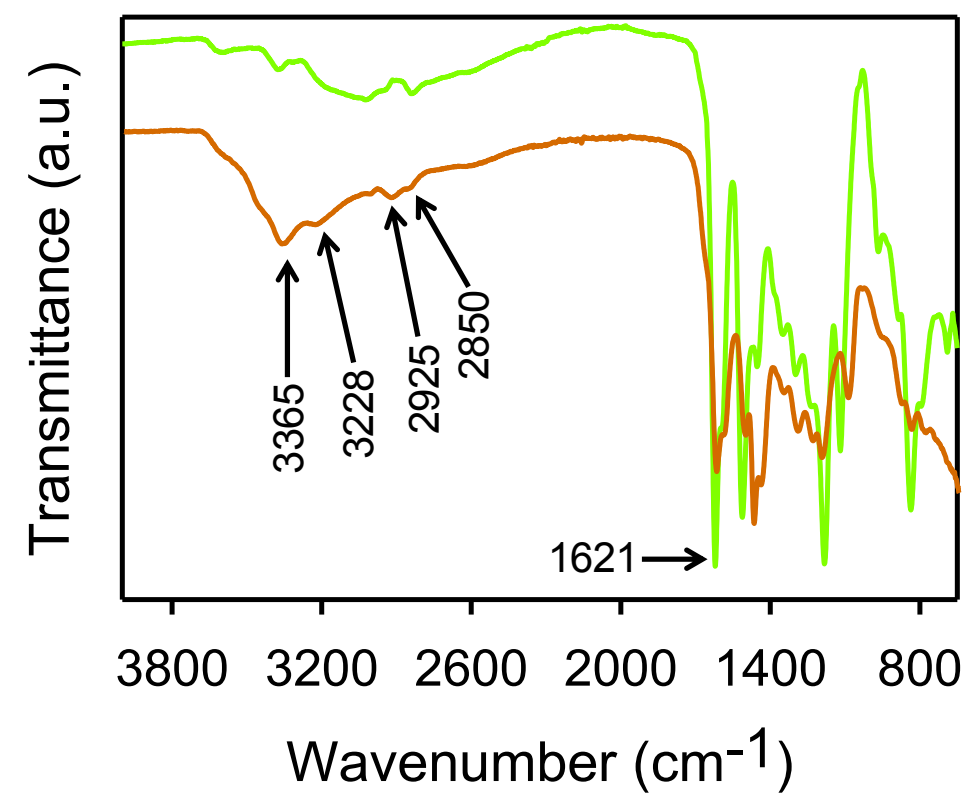

Figure S2. ATR-FTIR spectra of as-synthesized (at $30{ }^{\circ} \mathrm{C}$ ) RNS1 (green) and RNS1 after hydrothermal curing at $100{ }^{\circ} \mathrm{C}$ for $24 \mathrm{~h}$ (orange).

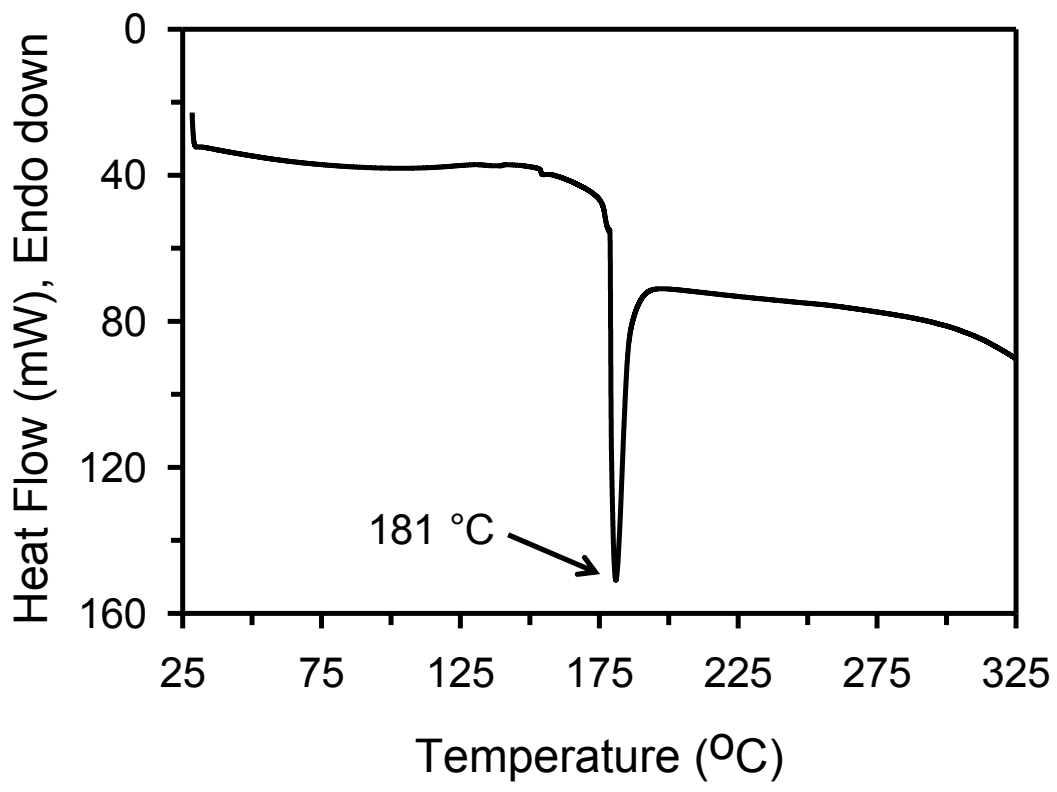

Figure S3. Differential scanning calorimetry (DSC) thermogram of RNS1 obtained at a scanning rate of $10{ }^{\circ} \mathrm{C} / \mathrm{min}$ under nitrogen. The peak at $181{ }^{\circ} \mathrm{C}$ has a heat of condensation of $139 \mathrm{~J} \mathrm{~g}^{-1}$ that arises from the polycondensation of the resin spheres. ${ }^{\mathrm{S} 3}$ 

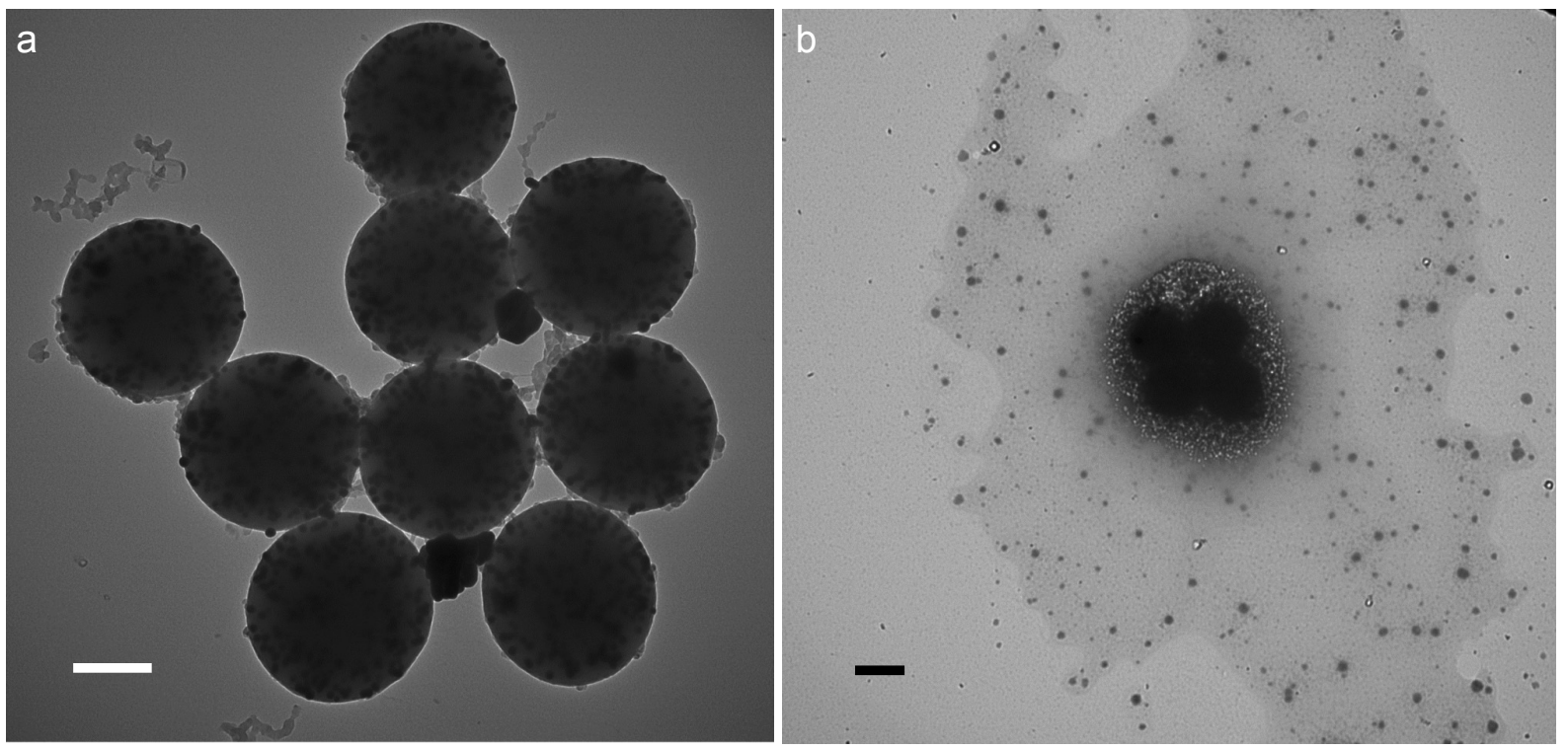

Figure S4. TEM images of the samples prepared by addition of $\mathrm{HAuCl}_{4}$ to an aqueous dispersion of resin spheres (before purification by washing) that still contained unreacted reagents. (a) TEM image of the resin spheres obtained after centrifugation. Scale bar, $200 \mathrm{~nm}$. (b) TEM image obtained by drop casting the supernatant solution on the TEM grid showing polydisperse gold nanoparticles with a few nanospheres that escaped precipitation during centrifugation and remained in the supernatant. Scale bar, $500 \mathrm{~nm}$. 


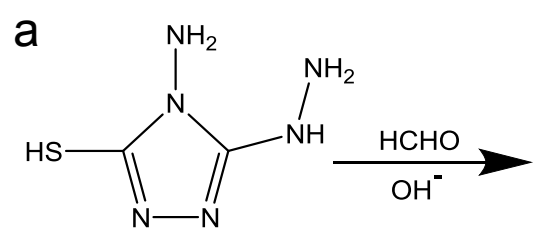

colorless

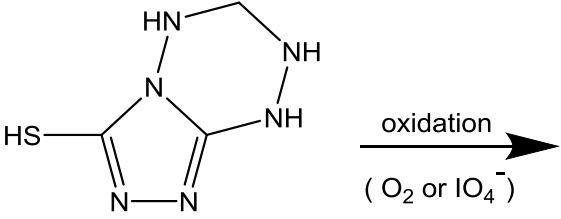

$\left(\mathrm{O}_{2}\right.$ or $\left.1 \mathrm{OO}_{4}^{-}\right)$<smiles>Sc1nnc2nncnn12</smiles>

purple

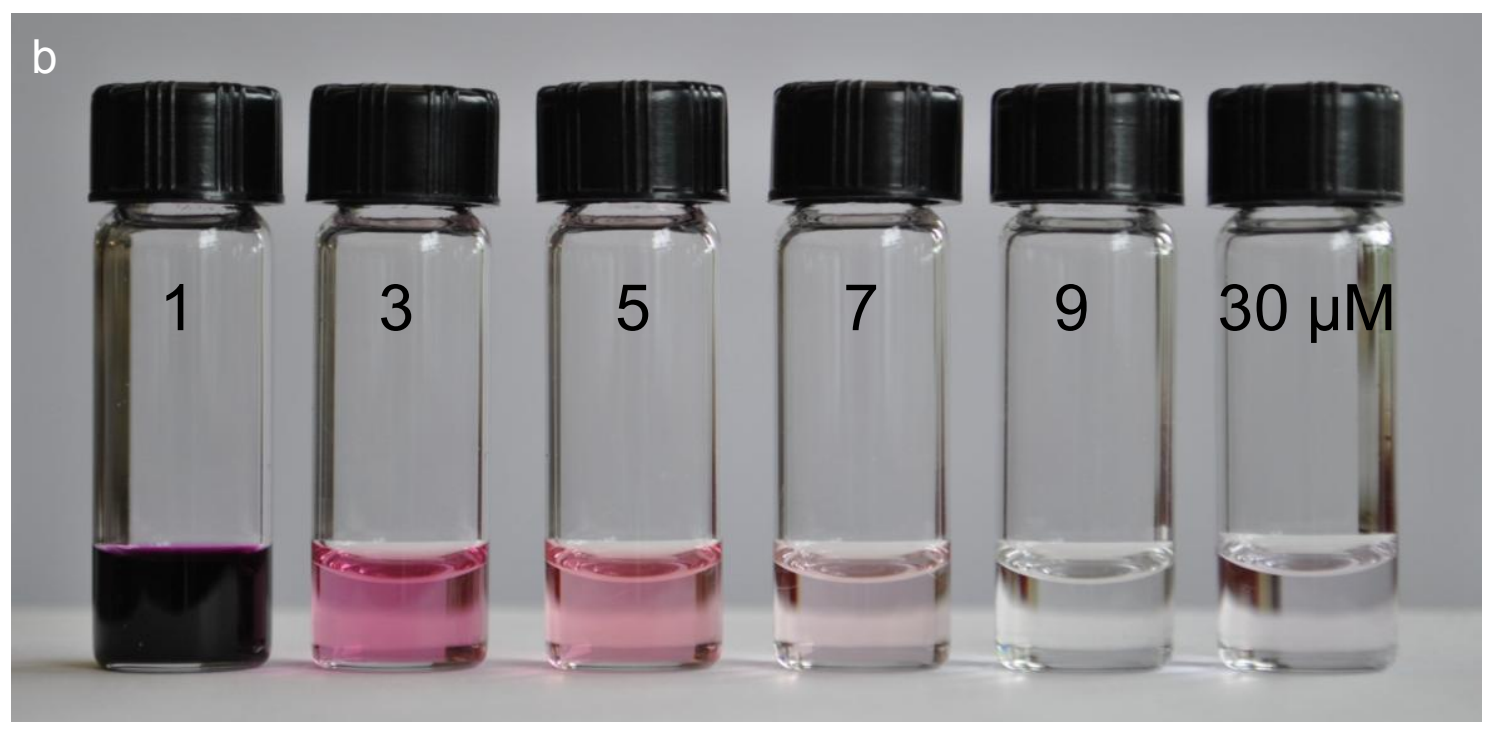

Figure S5. (a) Reaction scheme for detection of formaldehyde. Formaldehyde is first condensed with Purpald (4-amino-3-hydrazino-5-mercapto-1,2,4-triazole) to form an intermediate that is subsequently oxidized to yield the colored product. (b) Photographs showing the presence of formaldehyde in the washings. The numbers on the first five vials indicate how many times the sample had been centrifuged and washed with distilled water before the supernatant was isolated (e.g., vial " 3 " means the supernatant after the resin nanosphere sample was centrifuged, isolated, washed with distilled water, sonicated, centrifuged, isolated, washed with distilled water, sonicated, and centrifuged). The vial on the right shows the color after performing the same test on a standard solution of $30 \mu \mathrm{M}$ formaldehyde. 

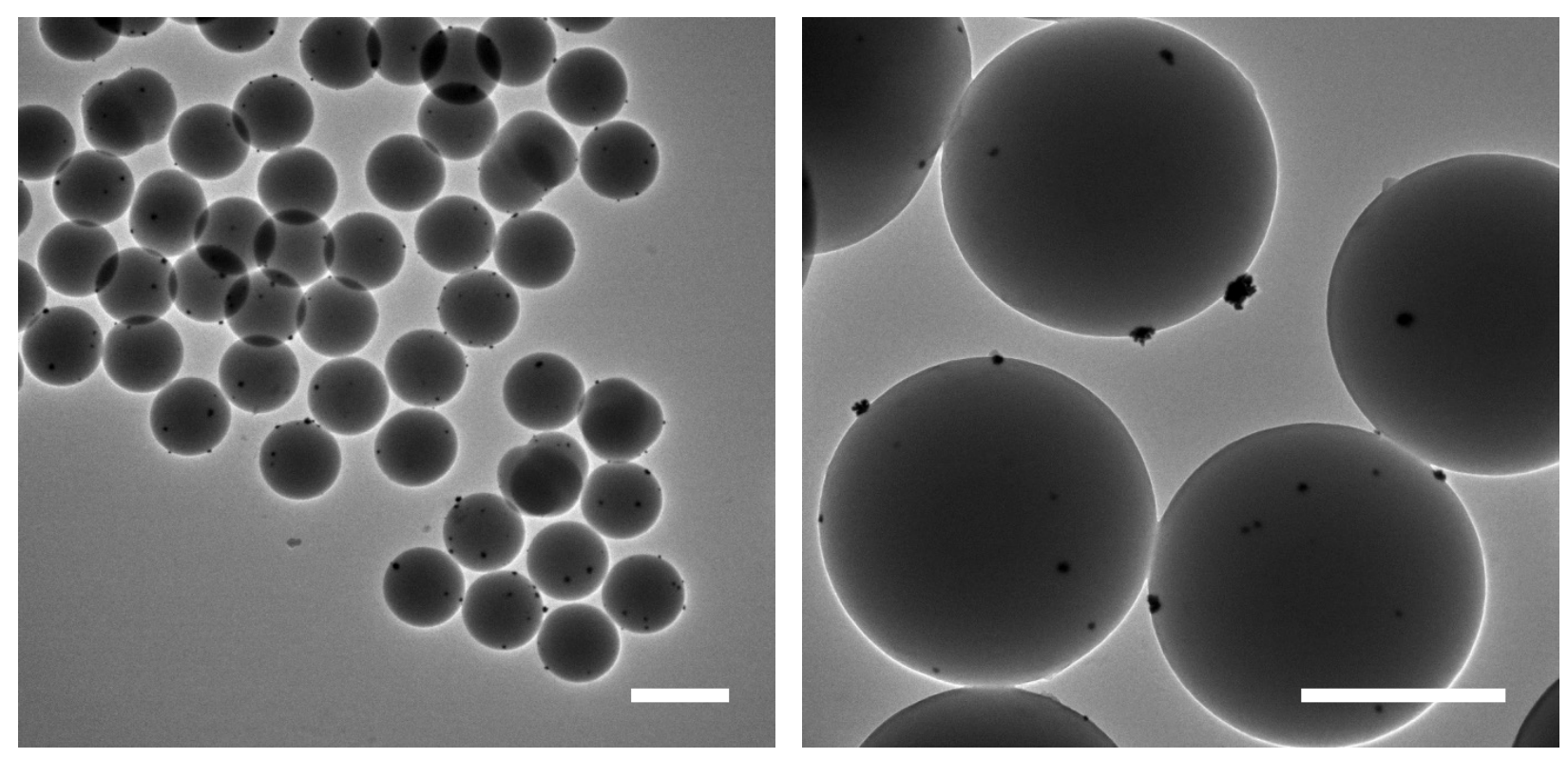

Figure S6. TEM images at two different magnifications of the sample prepared with simultaneous addition of gold and resin precursors during synthesis of the resin nanospheres. AuNPs are clearly visible on the surface of the spheres and there are no well-defined nanoparticles inside the spheres. Scale bar on the left image is $1 \mu \mathrm{m}$ and on the right image is $500 \mathrm{~nm}$. 

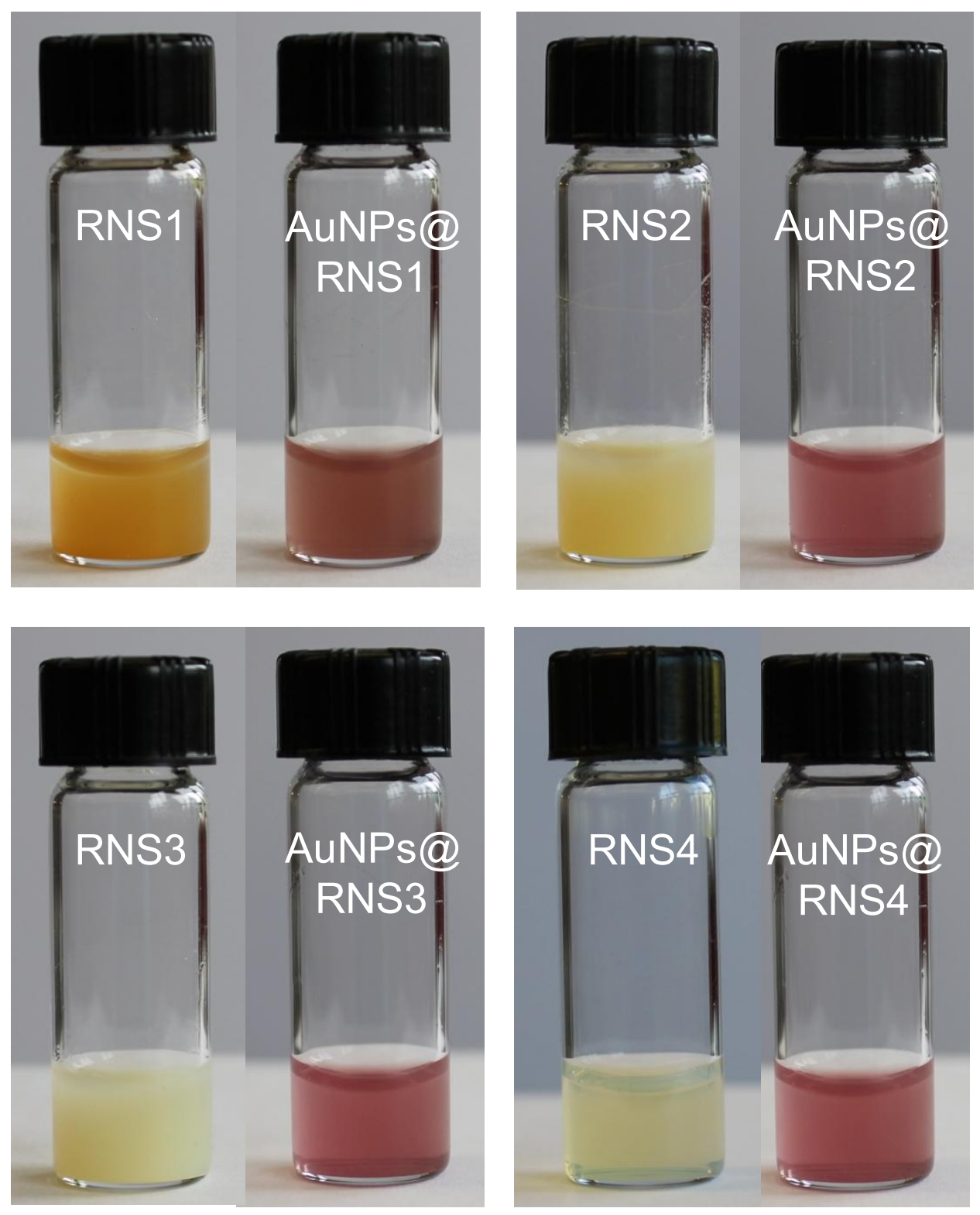

Figure S7. Photographs of RNS1 - RNS4 suspended in water before (left) and after gold addition (right). 

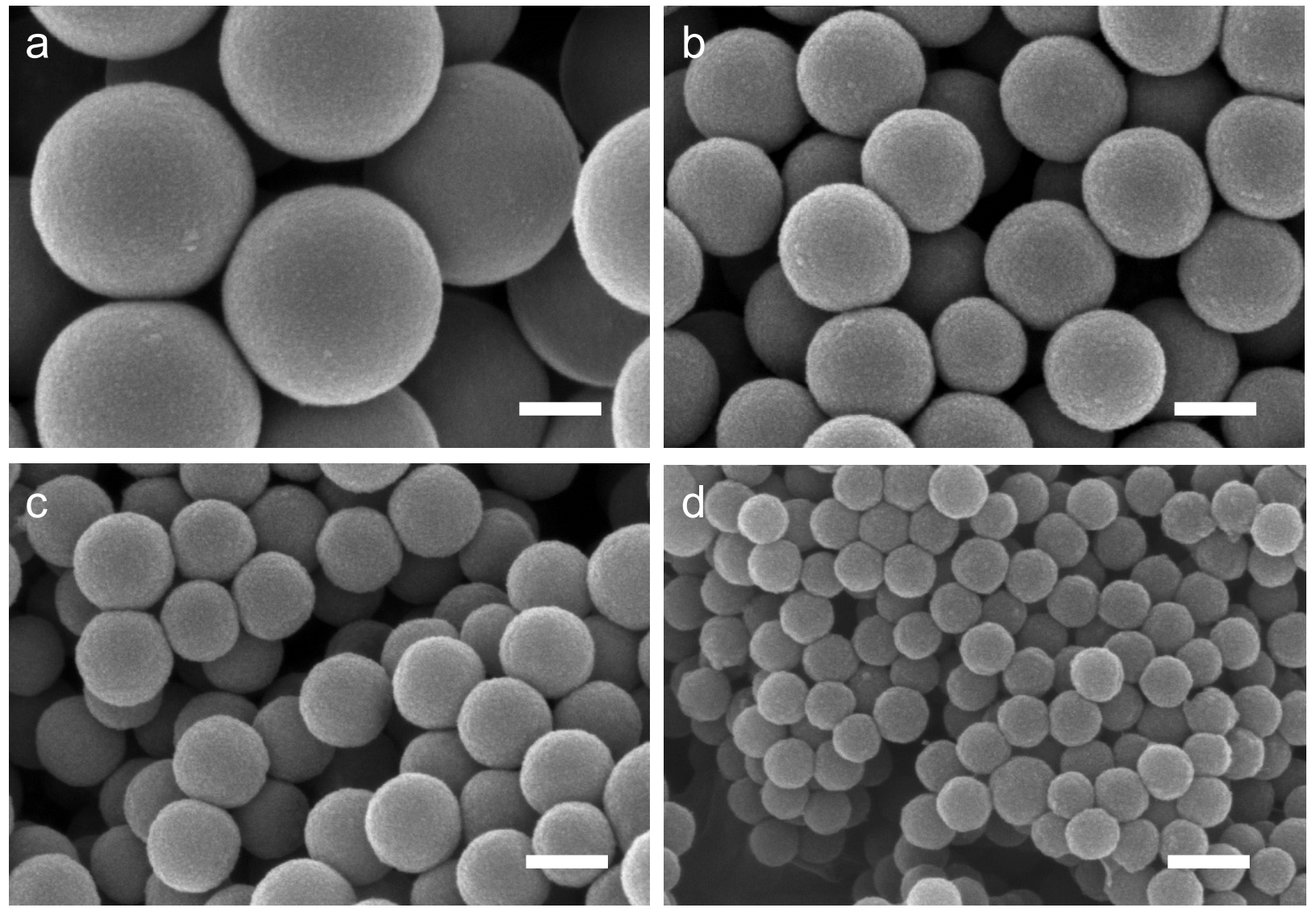

Figure S8. SEM images of (a) AuNPs@RNS1, (b) AuNPs@RNS2, (c) AuNPs@RNS3, and (d) AuNPs@RNS4 samples. Scale bars, 200 nm. 

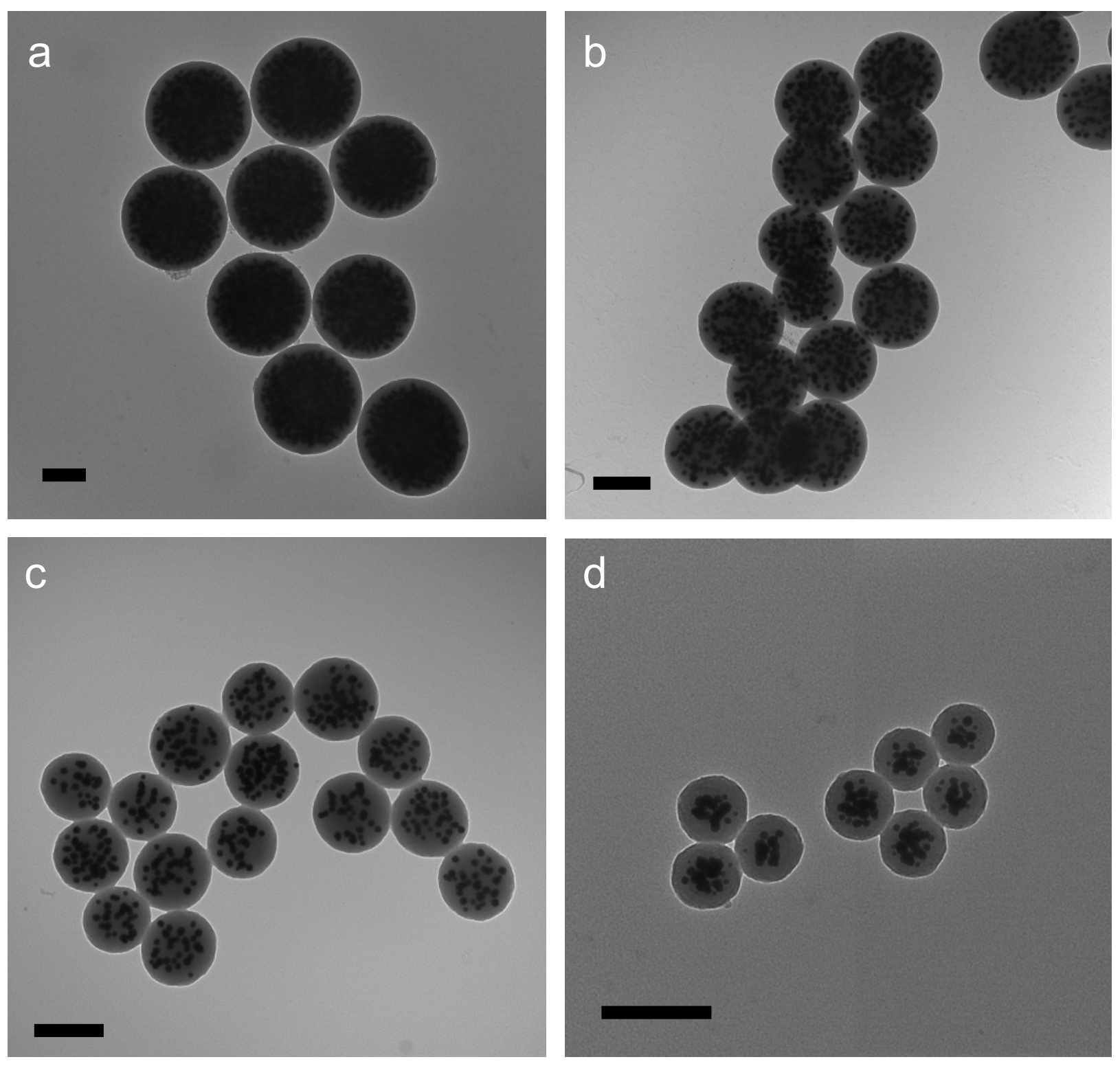

Figure S9. TEM images of (a) AuNPs@RNS1, (b) AuNPs@RNS2, (c) AuNPs@RNS3, and (d) AuNPs@RNS4 samples. Scale bars, 200 nm. 

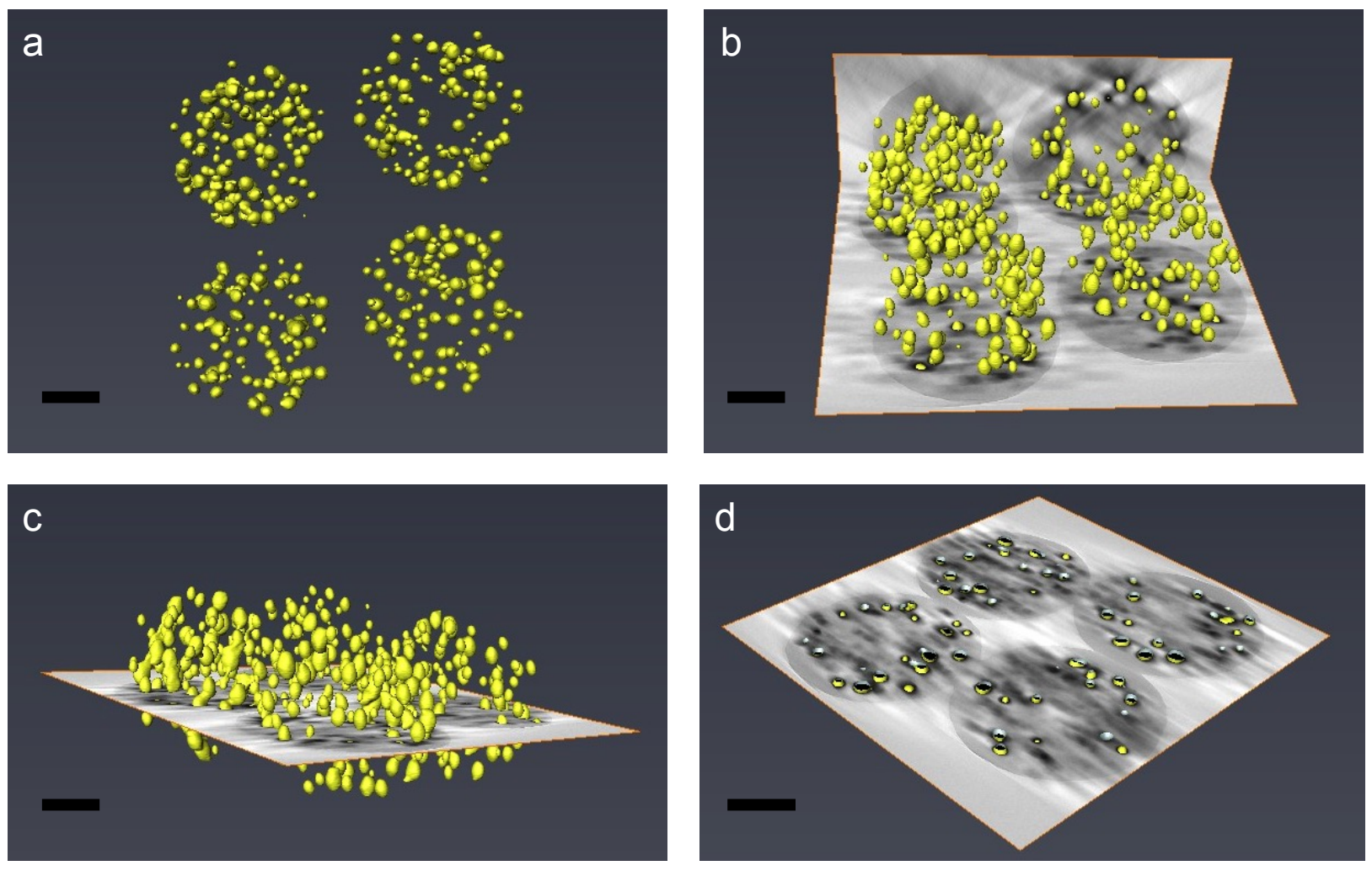

Figure S10. Electron tomography images of AuNPs@RNS2. Scale bars, 100 nm. 

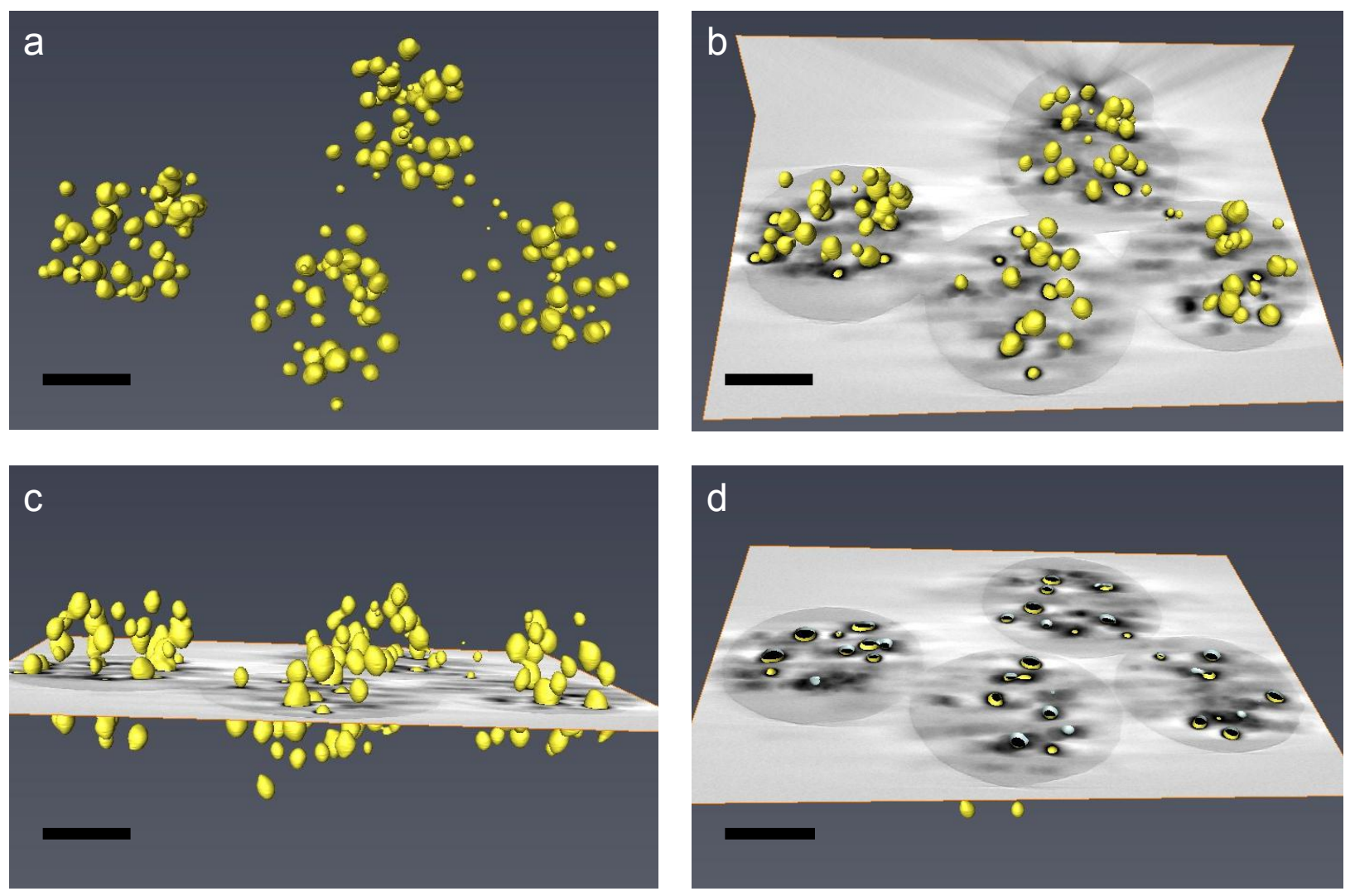

Figure S11. Electron tomography images of AuNPs@RNS3. Scale bars, 100 nm. 

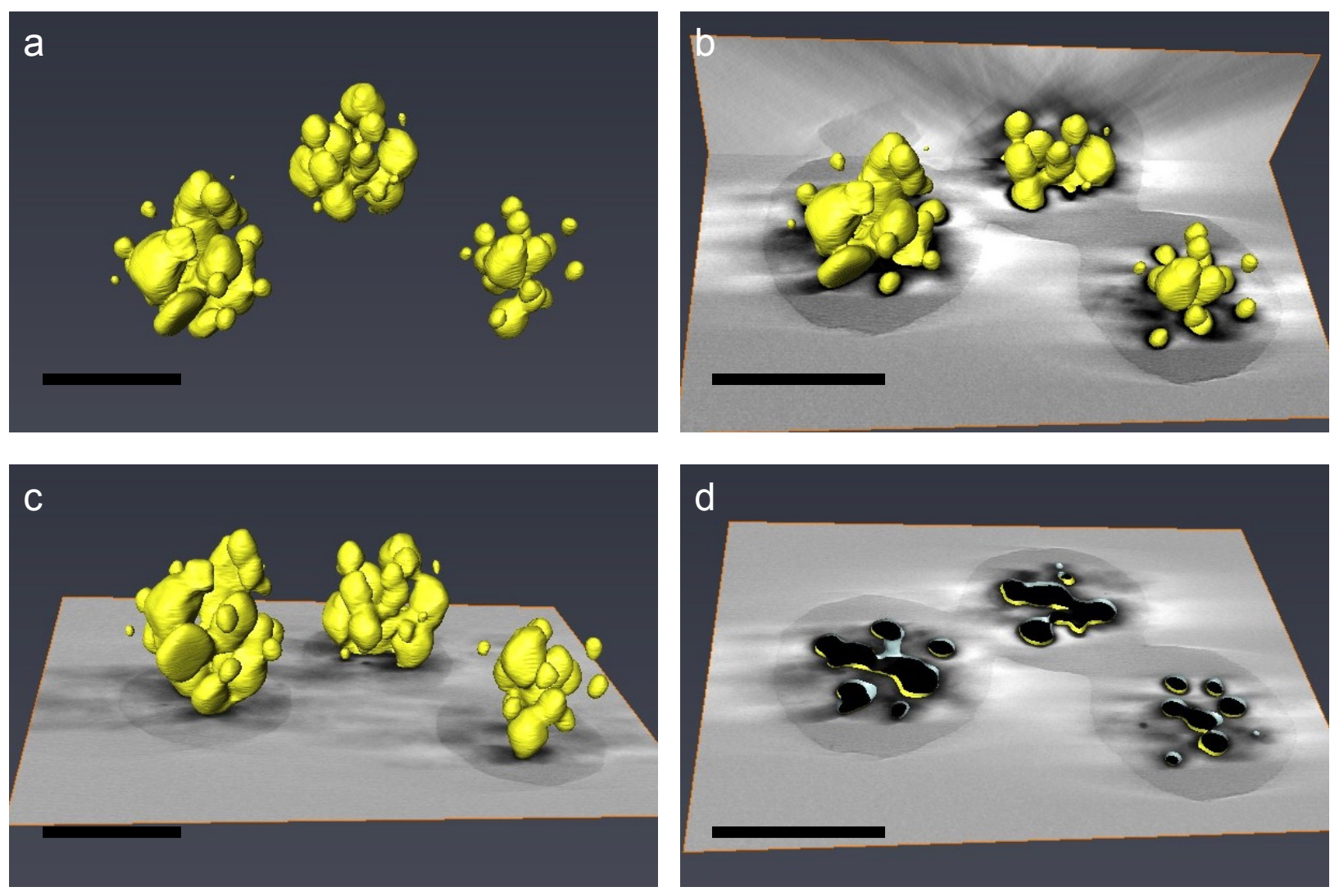

Figure S12. Electron tomography images of AuNPs@RNS4. Scale bars, 100 nm. 

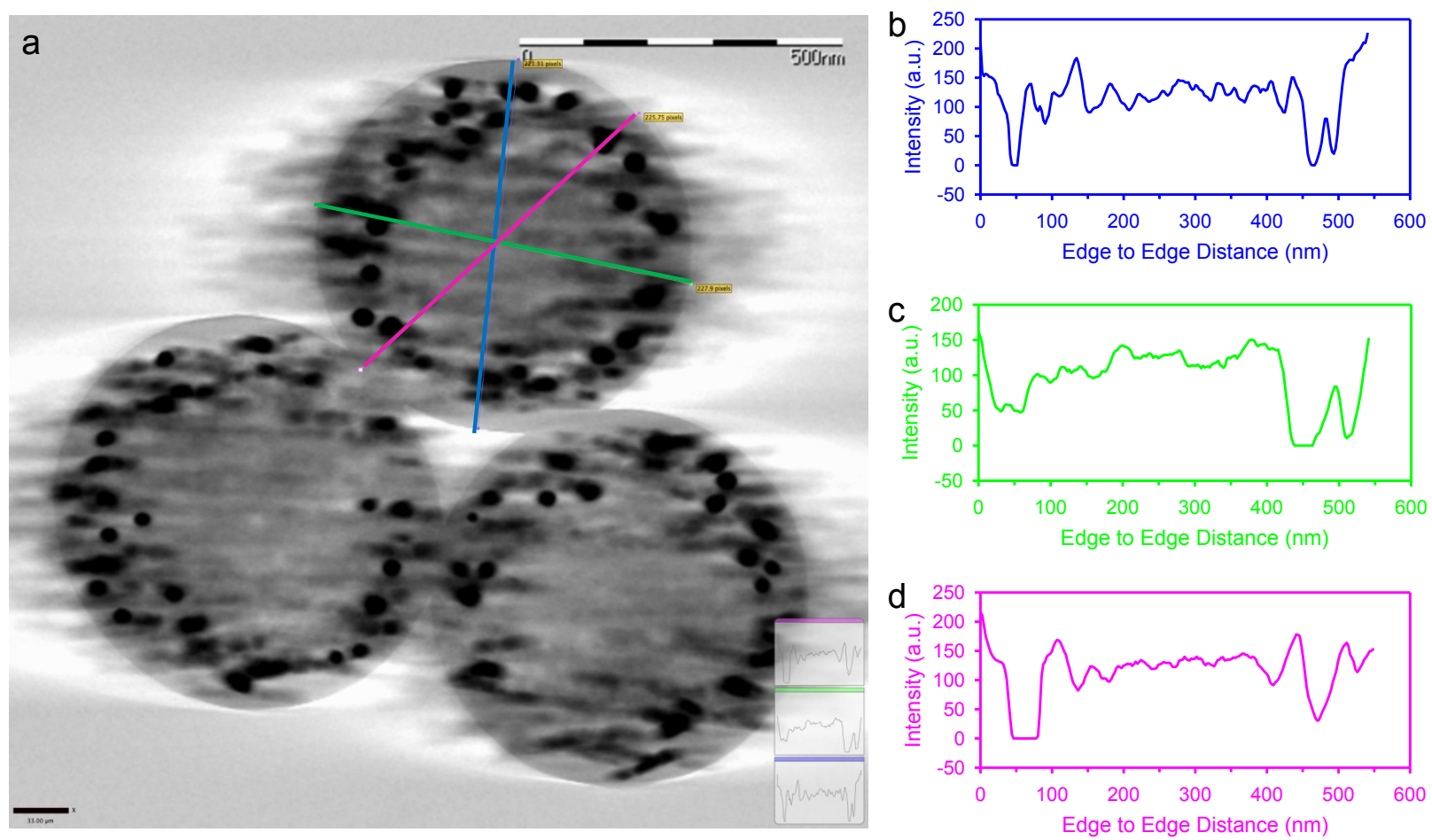

Figure S13. (a) Electron tomography image of AuNPs@RNS1 showing the cross-section. Density profile measurement along the blue line (b), green line (c), and pink line (d). 


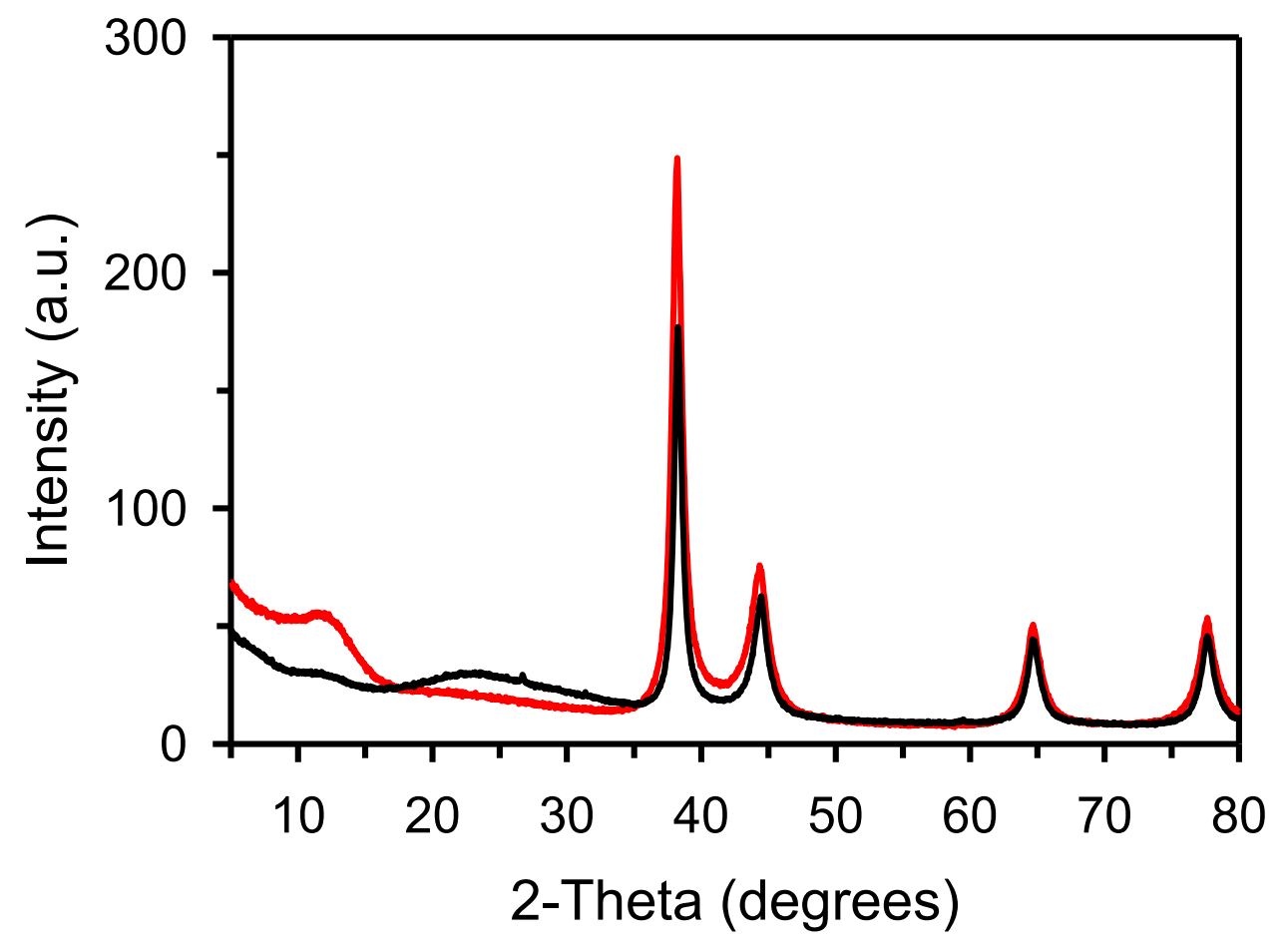

Figure S14. Powder X-ray diffraction (PXRD) traces of AuNPs@RNS1 (red) and AuNPs@CNS1 (black). 

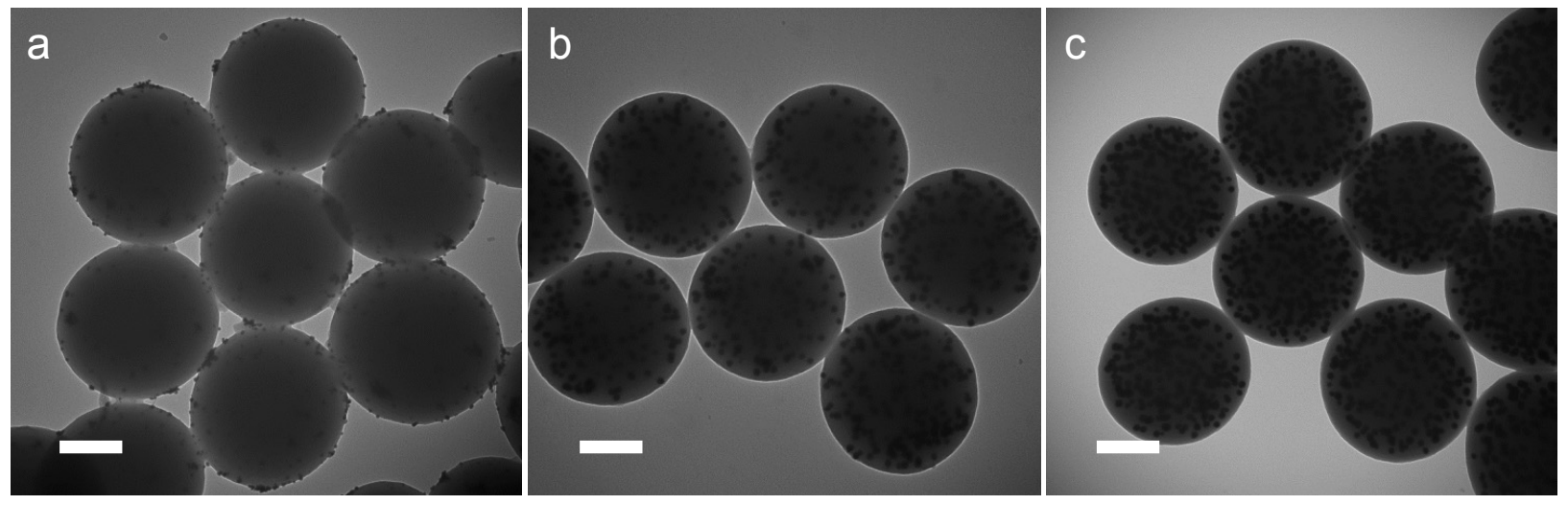

Figure S15. TEM images showing the effect of concentration of $\mathrm{HAuCl}_{4}$ on the morphology of the AuNPs@RNS1 composite materials. Samples were prepared by magnetic stirring of the RNS1 dispersion for $2 \mathrm{~h}$ at room temperature with varying concentration of $\mathrm{HAuCl}_{4}$ : (a) 0.1 $\mathrm{mM}$, (b) $0.5 \mathrm{mM}$, and (c) $1.0 \mathrm{mM}$. Scale bars, $200 \mathrm{~nm}$. 

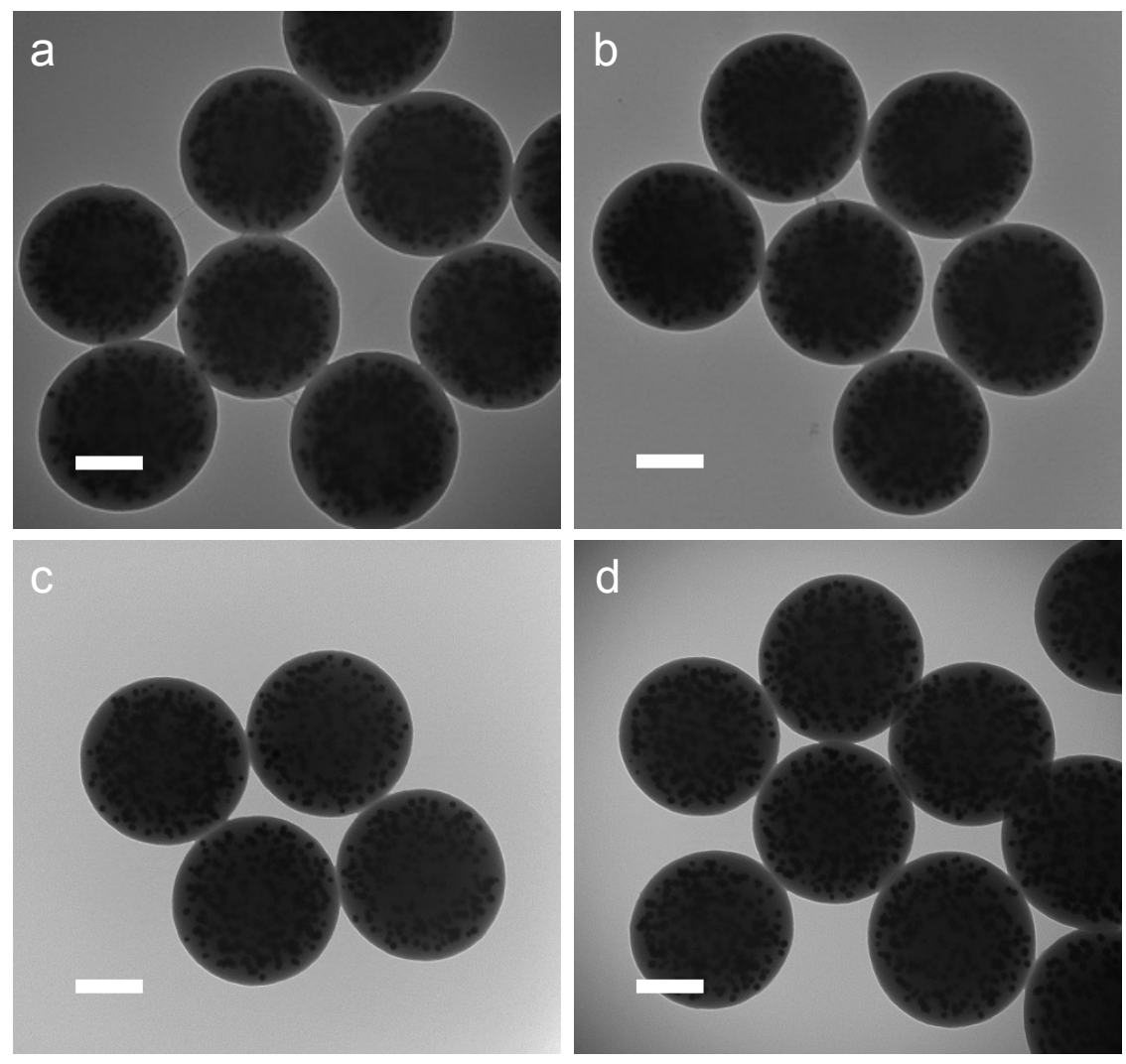

Figure S16. TEM images showing the effect of reaction time on the morphology of the AuNPs@RNS1 composite materials. Samples were prepared by magnetic stirring of the RNS1 dispersion containing $1.0 \mathrm{mM}$ of $\mathrm{HAuCl}_{4}$ at room temperature for different lengths of time: (a) $10 \mathrm{~min}$, (b) $30 \mathrm{~min}$, (c) $60 \mathrm{~min}$, and (d) $120 \mathrm{~min}$. Scale bars, $200 \mathrm{~nm}$. 


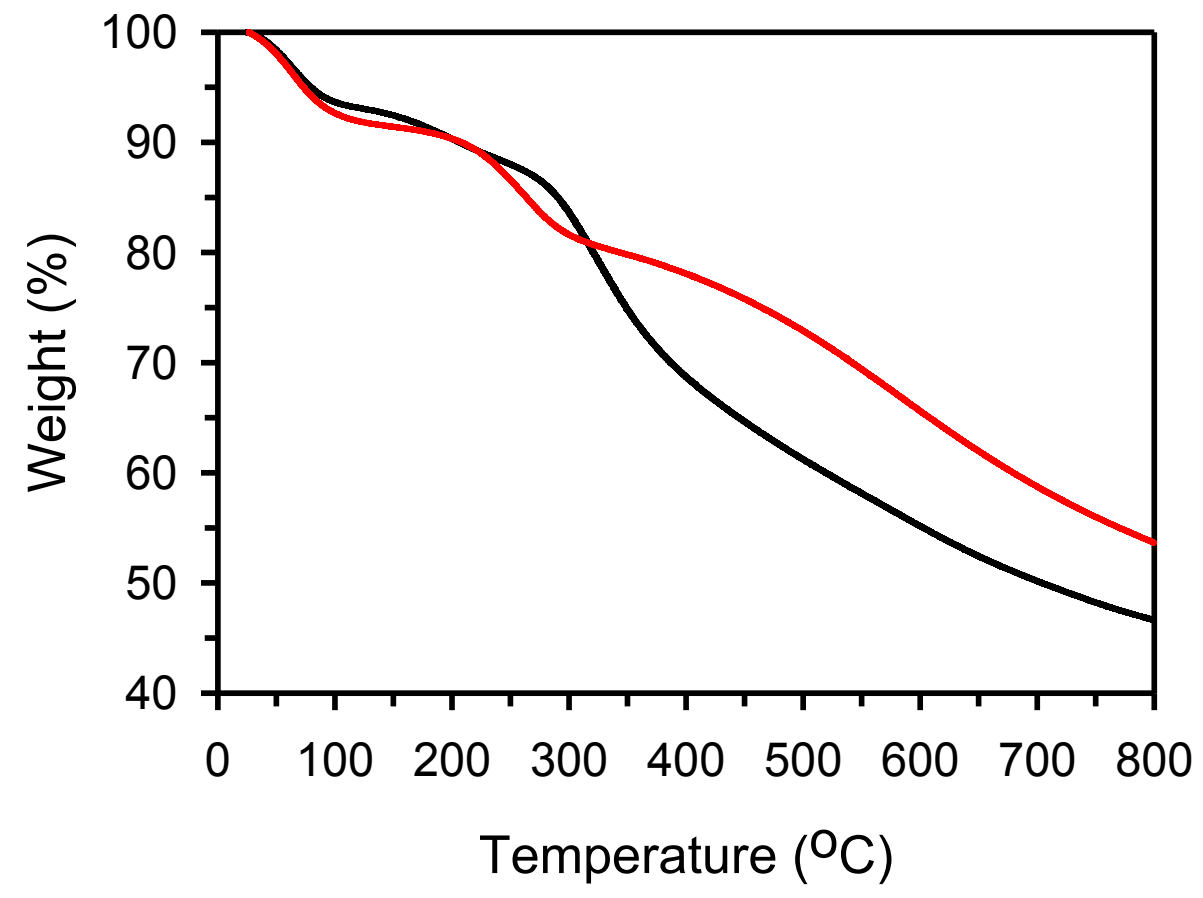

Figure S17. Thermogravimetric analysis of RNS1 (black) and AuNPs@RNS1 (red) samples obtained at a scan rate of $10^{\circ} \mathrm{C} / \mathrm{min}$ under nitrogen. 

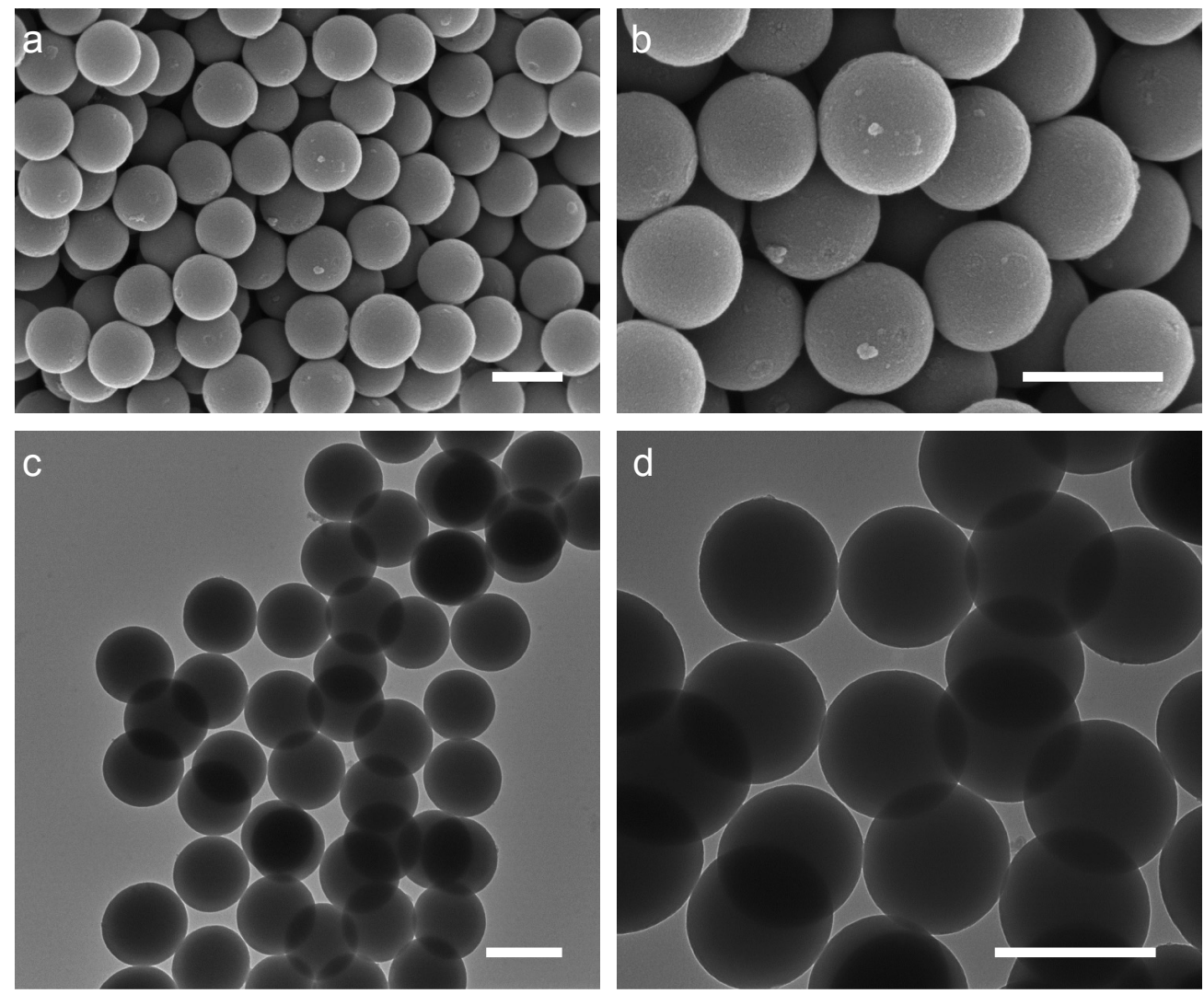

Figure S18. SEM (a, b) and TEM (c, d) images of CNS1. Scale bars, $500 \mathrm{~nm}$.

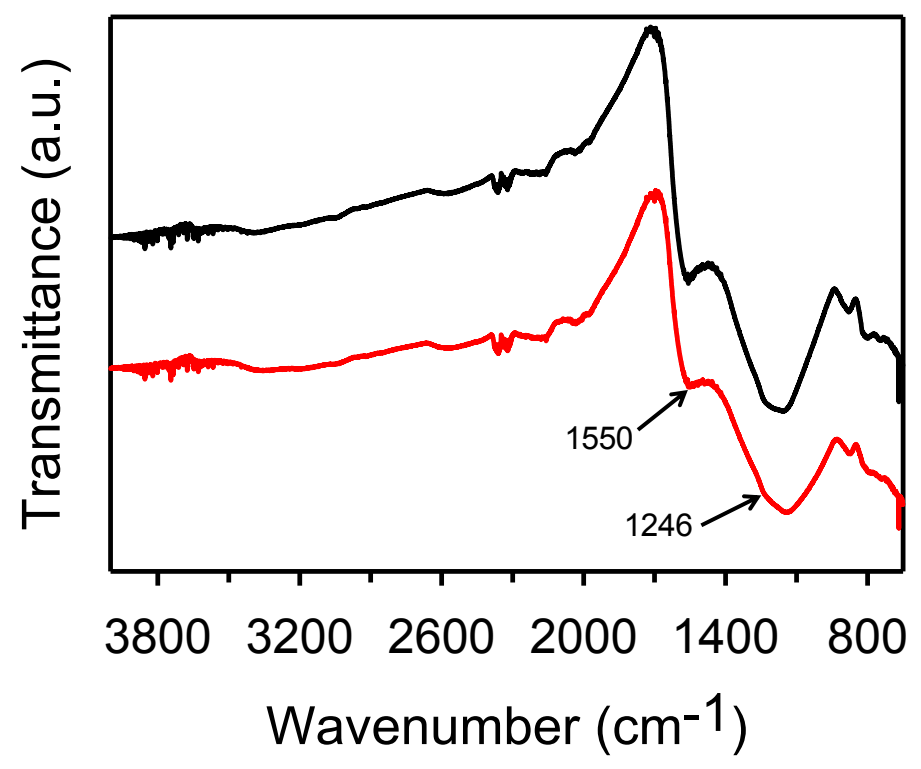

Figure S19. ATR-FTIR spectra of CNS1 (black) and AuNPs@CNS1 (red). 

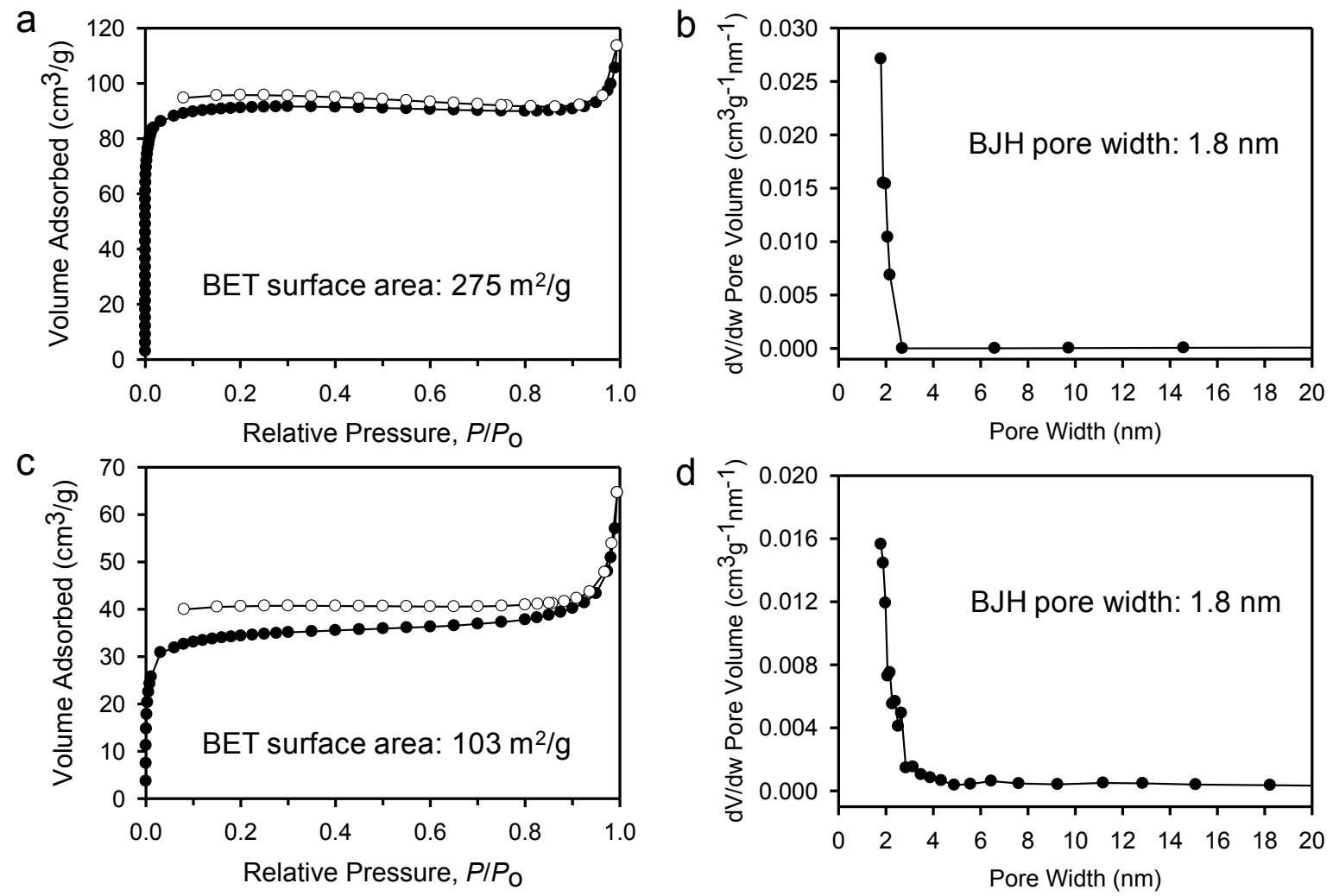

Figure S20. (a) Adsorption (solid circles) and desorption (open circles) isotherms of CNS1. (b) BJH pore size distribution of CNS1. (c) Adsorption (solid circles) and desorption (open circles) isotherms of AuNPs@CNS1. (d) BJH pore size distribution of AuNPs@CNS1.

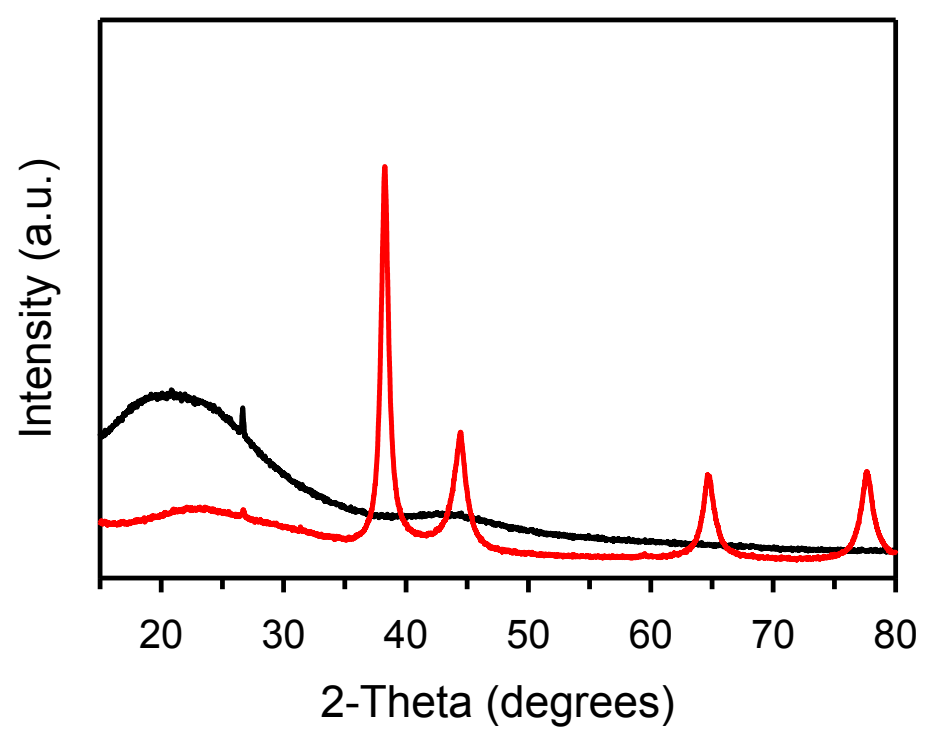

Figure S21. PXRD patterns of CNS1 (black) and AuNPs@CNS1 (red). 


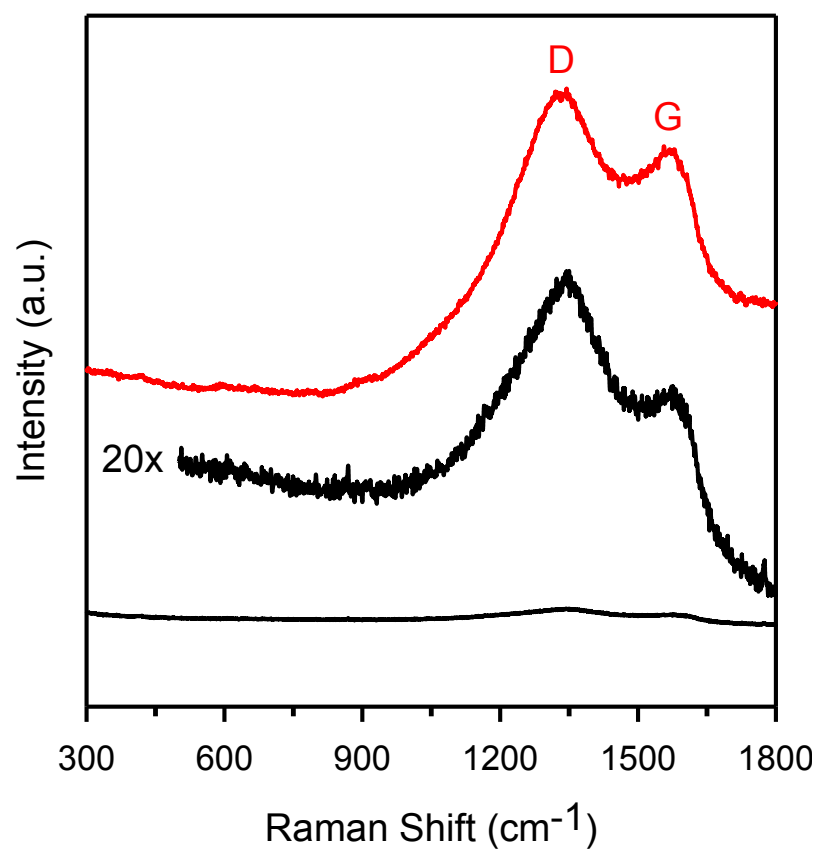

Figure S22. Raman spectra of CNS1 (black) and AuNPs@CNS1 (red). Spectra were plotted on absolute Y-axis scale. The bold black line is the spectrum of CNS1 that was enlarged by 20x on $\mathrm{Y}$-axis scale and superimposed above the original trace for clarity and better identification of the peaks.

Table S2. Results of elemental analysis.

\begin{tabular}{|c|c|c|c|}
\hline \multirow{2}{*}{ Samples } & \multicolumn{3}{|c|}{ Elements Determined } \\
\cline { 2 - 4 } & $\mathbf{\%}$ C & \%N & \%H \\
\hline RNS1 & 64.41 & 12.73 & 6.00 \\
\hline AuNPs@RNS1 & 43.13 & 8.29 & 4.24 \\
\hline RNS2 & 63.23 & 13.18 & 6.15 \\
\hline AuNPs@RNS2 & 43.03 & 8.33 & 4.00 \\
\hline RNS3 & 62.19 & 12.89 & 6.08 \\
\hline AuNPs@RNS3 & 42.62 & 8.33 & 4.08 \\
\hline RNS4 & 48.74 & 11.15 & 6.06 \\
\hline AuNPs@RNS4 & 40.10 & 7.71 & 3.87 \\
\hline CNS1 & 78.11 & 8.20 & 2.36 \\
\hline AuNPs@CNS1 & 40.64 & 3.97 & 1.56 \\
\hline
\end{tabular}




\section{References}

S1. Quesenberry, M. S.; Lee, Y. C. Anal. Biochem. 1996, 234, 50.

S2. Wang, L.; Zhou, C. L.; Chen, H. Q.; Chen, J. G.; Fu, J.; Ling, B. Analyst 2010, 135, 2139.

S3. Lee, Y.-K.; Kim, D.-J.; Kim, H.-J.; Hwang, T.-S.; Rafailovich, M.; Sokolov, J. J. Appl. Polym. Sci. 2003, 89, 2589. 


\section{Supporting Movie Captions}

Movie S1. Movie constructed from a series of TEM images captured with tilt angles ranging from -60 to $+60^{\circ}$ displaying AuNPs@RNS1 with total viewing angle of $120^{\circ}$.

Movie S2. Movie constructed from TEM images using the Serial Iterative Reconstruction Technique (SIRT) showing the scanned view of AuNPs@RNS1 at different heights along the zdirection (z-axis is defined in Fig. 3b). The first half of the movie shows the scanned view from the bottom to the top of the spheres and the second half shows the bounced-back view starting from the top and coming down to the bottom of the spheres.

Movie S3. Movie constructed from a series of TEM images captured with tilt angles ranging from -60 to $+60^{\circ}$ displaying AuNPs@RNS2 with total viewing angle of $120^{\circ}$.

Movie S4. Movie constructed from TEM images using the Serial Iterative Reconstruction Technique (SIRT) showing the scanned view of AuNPs@RNS2 at different heights along the zdirection. The first half of the movie shows the scanned view from the bottom to the top of the spheres and the second half shows the bounced-back view starting from the top and coming down to the bottom of the spheres.

Movie S5. Movie constructed from a series of TEM images captured with tilt angles ranging from -60 to $+60^{\circ}$ displaying AuNPs@RNS3 with total viewing angle of $120^{\circ}$.

Movie S6. Movie constructed from TEM images using the Serial Iterative Reconstruction Technique (SIRT) showing the scanned view of AuNPs@RNS3 at different heights along the zdirection. First half of the movie shows the scanned view from the bottom to the top of the spheres and the second half shows the bounced-back view starting from the top and coming down to the bottom of the spheres.

Movie S7. Movie constructed from a series of TEM images captured with tilt angles ranging from -60 to $+60^{\circ}$ displaying AuNPs@RNS4 with total viewing angle of $120^{\circ}$.

Movie S8. Movie constructed from TEM images using the Serial Iterative Reconstruction Technique (SIRT) showing the scanned view of AuNPs@RNS4 at different heights along the zdirection. The first half of the movie shows the scanned view from the bottom to the top of the spheres and the second half shows the bounced-back view starting from the top and coming down to the bottom of the spheres.

Movie S9. Movie constructed from a series of TEM images captured with tilt angles ranging from -60 to $+60^{\circ}$ displaying AuNPs@CNS1 with total viewing angle of $120^{\circ}$.

Movie S10. Movie constructed from TEM images using the Serial Iterative Reconstruction Technique (SIRT) showing the scanned view of AuNPs@CNS1 at different heights along the zdirection (z-axis is defined in Fig. 4c). First half of the movie shows the scanned view from the bottom to the top of the spheres and the second half shows the bounced-back view starting from the top and coming down to the bottom of the spheres. 